4. O. Szász, On the Gibbs phenomenon for Euler means, Acta Sci. Math. Szeged Part B vol. 12 (1950) pp. 107-111.

5. —, Gibbs phenomenon for Hausdorff means, Trans. Amer. Math. Soc. vol. 69 (1950) pp. 440-456.

6. A. Zygmund, Trigonometrical series, Warsaw, 1935.

National Taiwan University, Taiwan, China

\title{
A NEW CLASS OF INTEGRAL TRANSFORMS
}

\section{TA $L I^{1}$}

Introduction. In aerodynamics, a velocity potential is usually represented as a singular integral given by a sink and source distribution. The integrand contains a strength factor. For cones and slender bodies, this strength factor is determined by methods $[1]^{2}$ either graphical or numerical as suggested by Tsien [2], von Kármán and Moore [3], and other writers. An exact solution was believed to be too difficult, for an integral equation of the first kind does not, in general, admit a solution.

In deriving a solution of a certain aerodynamical problem, the writer was led to a much more general class of integral equations, each of which has, as its kernel, a Chebyshev polynomial of the first kind, divided by the square root of the difference of two squares. The writer was fortunate in having found the exact solution to each of these singular integral equations. The solution is given in the form of a singular integral involving a Chebyshev polynomial of the first kind. The application of these results to aerodynamics is immediate.

This paper is dedicated to Professor O. Perron, of Munich, on his 79th birthday, in appreciation of his achievement as a mathematician and for his success as a teacher.

Chebyshev polynomials of the first kind. The Chebyshev polynomial of the first kind and $n$th degree is denoted by $T_{n}(t)$. It is defined as the polynomial solution of the differential equation

$$
\left(x^{2}-1\right) y^{\prime \prime}(x)+x y^{\prime}(x)-n^{2} y(x)=0
$$

Presented to the Society, January 21, 1959; received by the editors March 30, 1959 and, in revised form, May 4, 1959 and May 25, 1959.

${ }^{1}$ Design Specialist, Dynamics Group, Convair-Astronautics, Convair Division of General Dynamics Corporation, San Diego, California; Lecturer, University of California, Los Angeles, California.

${ }^{2}$ Numbers in brackets indicate references at the end of this paper. 
with the initial condition $y(1)=1$. Writing

$$
y(x)=x^{\delta} z\left(x^{2}-1\right)
$$

where

we find, after letting

$$
\delta= \begin{cases}0 & \text { when } n \text { is even } \\ 1 & \text { when } n \text { is odd }\end{cases}
$$

$$
x^{2}-1=t,
$$

the following differential equation for $z(t)$ :

$$
4 t(t+1) z^{\prime \prime}+2[2(1+\delta) t+1] z^{\prime}-4\left[\frac{n}{2}\right]\left[\frac{n+1}{2}\right] z=0
$$

where $[\xi]$ denotes the positive integral part of $\xi$. The polynomial solution of (5) satisfying $z(0)=1$ is

$$
\begin{aligned}
& (t+1)^{-\delta / 2} T_{n}\left((t+1)^{1 / 2}\right) \\
& =\frac{\left[\frac{n}{2}\right] !}{\left[\frac{n-1}{2}\right] !} \sum_{k=0}^{[n / 2]} \frac{2^{2 k} \Gamma\left(\left[\frac{n-1}{2}\right]+k+1\right)}{\Gamma\left(\left[\frac{n}{2}\right]-k+1\right) \Gamma(2 k+1)} t^{k} .
\end{aligned}
$$

Replacing $t$ by $x^{2}-1$ in (6), one finds the following lemma:

Lemma 1. The Chebyshev polynomial $T_{n}(t)$ defined by the differential equation (1) and the condition $y(1)=1$ can be written in the form

$$
T_{n}(x)=\frac{\left[\frac{n}{2}\right] !}{\left[\frac{n-1}{2}\right] !} x^{\delta} \sum_{k=0}^{[n / 2]} \frac{2^{2 k}\left(\left[\frac{n-1}{2}\right]+k\right) !}{\left(\left[\frac{n}{2}\right]-k\right) !(2 k) !}\left(x^{2}-1\right)^{k}
$$

where $\delta$ is given by (3).

(7) is the expression of the Chebyshev polynomial most suitable for our discussion.

Integral equations and their solutions. A large class of integral equations, including those mentioned in the summary, can be brought to the form

$$
\int_{0}^{1} \frac{T_{n}(u / \sigma) y_{n}(u) d u}{\left(u^{2}-\sigma^{2}\right)^{1 / 2}}=f_{n}(\sigma), \quad \sigma \in I, n=0,1,2,3, \cdots
$$


where the integral is taken in the sense of Riemann, $I=\{\sigma: c \leqq \sigma \leqq 1\}$, $c>0$ a constant, and $f_{n}(\sigma)$ is defined on $I$. It is assumed that

(a) $f_{n}(1)=0$,

(b) $(d / d \sigma)\left[\sigma^{n} f_{n}(\sigma)\right]$ is piecewise continuous on $I$.

Condition (a) is necessary for $y_{n}(u)$ to remain finite on $I$. The same condition also implies that $(d / d \sigma)\left[\sigma^{n} f_{n}(\sigma)\right] \not \equiv 0$; for otherwise $f_{n}(\sigma)$ $=C \sigma^{-n}, C$ constant, contradicting (a).

The solution of $(8)$ is given by

$$
y_{n}(u)=-\frac{2}{\pi} \int_{u}^{1} \frac{T_{n-1}(u / v) d\left[v^{n} f_{n}(v)\right]}{v^{n-1}\left(v^{2}-u^{2}\right)^{1 / 2}}
$$

where in the case of $n=0, T_{-1}(x)$ is interpreted as $T_{1}(x)$ by virtue of the differential equation (1). A proof of the dual relations (8) and (9) will be given later.

We want to note in passing that the solution of

$$
\int_{0}^{1} \frac{y_{0}(u) d u}{\left(u^{2}-\sigma^{2}\right)^{1 / 2}}=f_{0}(\sigma)
$$

was given as

$$
y_{0}(u)=-\frac{2}{\pi} \frac{d}{d u} \int_{u}^{1} \frac{v f_{0}(v) d v}{\left(v^{2}-u^{2}\right)^{1 / 2}}
$$

by $M$. Bôcher [4]. In case $f_{0}(1)=0$, this can be reduced to the form

$$
y_{0}(u)=-\frac{2}{\pi} \int_{u}^{1} \frac{v T_{1}(u / v) d f_{0}(v)}{\left(v^{2}-u^{2}\right)^{1 / 2}}
$$

by writing

$$
\frac{v}{\left(v^{2}-u^{2}\right)^{1 / 2}} d v=d\left(v^{2}-u^{2}\right)^{1 / 2}
$$

and by integrating by parts. The differentiation with respect to $u$ is then carried out under the integral sign.

It should be noted that condition (a) does not necessarily impose a restriction on $f_{n}(\sigma)$. For instance, if $f_{0}(1)=C \neq 0$, there is no loss of generality assuming $C=1$. The solution of

$$
\int_{\sigma}^{1} \frac{z_{0}(u) d u}{\left(u^{2}-\sigma^{2}\right)^{1 / 2}}=1
$$

is evidently 


$$
z_{0}(u)=\frac{2}{\pi} \frac{u}{\left(1-u^{2}\right)^{1 / 2}}
$$

Writing

$$
f_{0}(\sigma)=1+f_{0}^{*}(\sigma)
$$

the solution of

$$
\int_{0}^{1} \frac{y_{0}(u) d u}{\left(u^{2}-\sigma^{2}\right)^{1 / 2}}=f_{0}(\sigma)
$$

is obtained as

$$
y_{0}(u)=\frac{2 u}{\pi}\left[\frac{1}{\left(1-u^{2}\right)^{1 / 2}}-\int_{u}^{1} \frac{d f_{0}^{*}(v)}{\left(v^{2}-u^{2}\right)^{1 / 2}}\right] .
$$

A treatment of the general case will be given in a separate paper.

Some relations between binomial coefficients. As a preparation for the proof of the dual relations (8) and (9), we have to establish some useful relations between the binomial coefficients. Let us consider the expression

$$
\begin{aligned}
g_{\rho} & =\left\{\frac{d^{\rho}}{d x^{\rho}}\left[x^{[(n-1) / 2]}(1-x)^{[n / 2]}\right]\right\}_{x=1} \\
& \times\left\{\frac{d^{[(n-2) / 2]+[(n-1) / 2]-\rho}}{d y^{[(n-2) / 2]+[(n-1) / 2]-\rho}}\left[y^{[(n-2) / 2]}(1-y)^{[(n-1) / 2]}\right]\right\}_{y=1} .
\end{aligned}
$$

It is clear that

$$
g_{\rho}=0 \quad \text { for } \rho=0,1,2, \cdots,[n / 2]-1 .
$$

In case $\rho=[n / 2]+\nu, \nu \geqq 0$, we find

$$
\left[\frac{n-2}{2}\right]+\left[\frac{n-1}{2}\right]-\rho=\left[\frac{n-1}{2}\right]-\nu-1
$$

so that one has

$$
\begin{aligned}
g_{\rho} & =(-1)^{[n / 2]}\left[\frac{n}{2}\right] !\left\{\frac{d^{\nu}}{d x^{\nu}} x^{[(n-1) / 2]}\right\}_{x=1} \\
& \times\left\{\frac{d^{[(n-1) / 2]-\nu-1}}{d y^{[(n-1) / 2]-\nu-1}}\left[y^{[(n-2) / 2]}(1-y)^{[(n-1) / 2]}\right]\right\}_{y=1}=0
\end{aligned}
$$

for $0 \leqq \nu<[(n-1) / 2]$

On the other hand, if we expand both $(1-x)^{[n / 2]}$ and $(1-y)^{[(n-1) / 2]}$ 
by binomial theorem and then differentiate the results, we will obtain:

$$
\begin{gathered}
g_{\rho}=\left[\frac{n}{2}\right] !\left[\frac{n-1}{2}\right] ! \sum_{k=0}^{[n / 2]} \frac{(-1)^{k}\left(\left[\frac{n-1}{2}\right]+k\right) !}{\left(\left[\frac{n}{2}\right]-k\right) ! k !\left(\left[\frac{n-1}{2}\right]+k-\rho\right) !} \\
\times \sum_{\mu=0}^{[(n-1) / 2]} \frac{(-1)^{\mu}\left(\left[\frac{n-2}{2}\right]+\mu\right) !}{\left(\left[\frac{n-1}{2}\right]-\mu\right) ! \mu !\left(\mu+\rho-\left[\frac{n-1}{2}\right]\right) !} \cdot
\end{gathered}
$$

Comparing this with (11) and (13), one finds the following useful relations:

Lemma 2. Let $n$ be an integer $\geqq 2$. Then

$$
\begin{aligned}
& \sum_{k=0}^{[n / 2]} \frac{(-1)^{k}\left(\left[\frac{n-1}{2}\right]+k\right) !}{\left(\left[\frac{n}{2}\right]-k\right) ! k !\left(\left[\frac{n-1}{2}\right]+k-\rho\right) !} \\
& \quad \times \sum_{\mu=0}^{[(n-1) / 2]} \frac{(-1)^{\mu}\left(\left[\frac{n-2}{2}\right]+\mu\right) !}{\left(\left[\frac{n-1}{2}\right]-\mu\right) ! \mu !\left(\mu+\rho-\left[\frac{n-1}{2}\right]\right) !}=0
\end{aligned}
$$

for $\rho=0,1,2,3, \cdots,[n / 2]+[(n-1) / 2]-1$, where for every $\rho$ only one of the factors is zero.

In addition to Lemma 2, one will find that it is useful to prove

LEMmA 3. Let $n$ be an integer $\geqq 2$, then we have

$$
\frac{\left(\left[\frac{n}{2}\right]+\left[\frac{n-1}{2}\right]\right) !}{\left[\frac{n-1}{2}\right] !\left[\frac{n-2}{2}\right] !}
$$

$$
\times \sum_{\mu=0}^{[(n-1) / 2]} \frac{(-1)^{\mu}\left[\frac{n-1}{2}\right] !}{\left(\left[\frac{n-1}{2}\right]+\mu\right)\left(\left[\frac{n-1}{2}\right]-\mu\right) ! \mu !}=1 .
$$


For proof of (16) one considers the expression

$$
I=\int_{0}^{1} x^{[n / 2]-1}(1-x)^{[(n-1) / 2]} d x
$$

which is $[(n-1) / 2] ![(n-2) / 2] ! /([n / 2]+[(n-1) / 2]) !$, when considered as an Euler's integral and is identical to the left hand side of (16) when $(1-x)^{[(n-1) / 2]}$ is expanded before integration. This completes the proof of (16).

Proof of the dual relations. On ground of conditions (a) and (b), it is obvious that the integral in (9) exists, and that the double integral:

$$
I=-\frac{2}{\pi} \int_{0}^{1} \frac{T_{n}(u / \sigma)}{\left(u^{2}-\sigma^{2}\right)^{1 / 2}} \int_{u}^{1} \frac{T_{n-1}(u / v) d\left[v^{n} f_{n}(v)\right]}{v^{n-1}\left(v^{2}-u^{2}\right)^{1 / 2}} d u,
$$

obtained by directly substituting (9) in (8), is convergent. This double integral can be written as

$$
I=-\frac{2}{\pi} \lim _{\epsilon \rightarrow 0} \int_{\sigma+\epsilon}^{1-\epsilon} \int_{u+\epsilon}^{1} \frac{T_{n}(u / \sigma) T_{n-1}(u / v) d\left[v^{n} f_{n}(v)\right]}{v^{n-1}\left(\left(u^{2}-\sigma^{2}\right)\left(v^{2}-u^{2}\right)\right)^{1 / 2}} d u .
$$

Since the above integrand remains finite and has at most a finite number of points of discontinuity in the region

$$
R:\left\{\begin{array}{l}
u+\epsilon \leqq v \leqq 1, \\
\sigma+\epsilon \leqq u \leqq 1,
\end{array}\right.
$$

it is justified to interchange the order of integration in $R$. Thus we obtain

$$
I=-\frac{2}{\pi} \lim _{\epsilon \rightarrow 0} \int_{\sigma+2 \epsilon}^{1} \frac{d\left[v^{n} f_{n}(v)\right]}{v^{n-1}} \int_{\sigma+\epsilon}^{v-\epsilon} \frac{T_{n}(u / \sigma) T_{n-1}(u / v) d u}{\left(\left(u^{2}-\sigma^{2}\right)\left(v^{2}-u^{2}\right)\right)^{1 / 2}} .
$$

Because $T_{n}(u / \sigma) T_{n-1}(u / v)$ is continuous and finite in the interval $v \leqq u \leqq \sigma$ and

$$
\int_{\sigma}^{v} \frac{d u^{2}}{\left(\left(u^{2}-\sigma^{2}\right)\left(v^{2}-u^{2}\right)\right)^{1 / 2}}=\pi
$$

the integral

$$
\int_{\sigma}^{v} \frac{T_{n}(u / \sigma) T_{n-1}(u / v) d u}{\left(\left(u^{2}-\sigma^{2}\right)\left(v^{2}-u^{2}\right)\right)^{1 / 2}}
$$

exists; so that one can write 


$$
I=-\frac{2}{\pi} \int_{0}^{1} \frac{d\left[v^{n} f_{n}(v)\right]}{v^{n-1}} \int_{\sigma}^{v} \frac{T_{n}(u / \sigma) T_{n-1}(u / v) d u}{\left(\left(u^{2}-\sigma^{2}\right)\left(v^{2}-u^{2}\right)\right)^{1 / 2}} .
$$

From Lemma 1 we have

$$
\begin{aligned}
& T_{n}\left(\frac{u}{\sigma}\right) T_{n-1}\left(\frac{u}{v}\right)=\frac{\left[\frac{n}{2}\right] !}{\left[\frac{n-2}{2}\right] !} \frac{u}{v}\left(\frac{v}{\sigma}\right)^{\delta} \\
& \times \sum_{k=0}^{[n / 2]} \sum_{\mu=0}^{[(n-1) / 2]}(-1)^{\mu} \frac{2^{2(k+\mu)}\left(\left[\frac{n-1}{2}\right]+k\right) !\left(\left[\frac{n-2}{2}\right]+\mu\right) !}{\left(\left[\frac{n}{2}\right]-k\right) !(2 k) !\left(\left[\frac{n-1}{2}\right]-\mu\right) !(2 \mu) !} \\
& \times \frac{1}{\sigma^{2 k}} \frac{1}{v^{2 \mu}}\left(u^{2}-\sigma^{2}\right)^{k}\left(v^{2}-u^{2}\right)^{\mu} .
\end{aligned}
$$

Substituting (19) in (18), writing $\left(u^{2}-\sigma^{2}\right)=\left(v^{2}-\sigma^{2}\right) x$, and making use of the following Euler's integral of the first kind:

$$
\int_{\sigma}^{v}\left(u^{2}-\sigma^{2}\right)^{k-1 / 2}\left(v^{2}-u^{2}\right)^{\mu-1 / 2} d u^{2}=\pi \frac{(2 k) !(2 \mu) !\left(v^{2}-\sigma^{2}\right)^{k+\mu}}{2^{2 k} 2^{2 \mu} k ! \mu !(k+\mu) !}
$$

(18) reduces to

$$
I=-\int_{0}^{1} \frac{d\left[v^{n} f_{n}(v)\right]}{v^{n}}\left(\frac{v}{\sigma}\right)^{\delta} B(t),
$$

where $t=v^{2} / \sigma^{2}$ and

$$
B(t)=\frac{\left[\frac{n}{2}\right] !}{\left[\frac{n-2}{2}\right] !}
$$

$\cdot \sum_{k=0}^{[n / 2]} \sum_{\mu=0}^{[(n-1) / 2]} \frac{(-1)^{\mu}\left(\left[\frac{n-1}{2}\right]+k\right) !\left(\left[\frac{n-2}{2}\right]+\mu\right) !(t-1)^{k+\mu} t^{-\mu}}{\left(\left[\frac{n}{2}\right]-k\right) !\left(\left[\frac{n-1}{2}\right]-\mu\right) ! k ! \mu !(k+\mu) !} \cdot$

The dual relations will be established if we can prove that

$$
t^{[(n-1) / 2]} B(t)=t^{[n / 2]+[(n-1) / 2]} .
$$


To prove (23) we expand $(t-1)^{k+\mu}$ according to the binomial theorem. This results in

$$
\begin{aligned}
& =\frac{\left[\frac{n}{2}\right] !}{\left[\frac{n-2}{2}\right] !} \sum_{k=0}^{t^{[(n-1) / 2]} B(t)} \frac{\left(\left[\frac{n-1}{2}\right]+k\right) !}{\left(\left[\frac{n}{2}\right]-k\right) ! k !} \sum_{\mu=0}^{[(n-1) / 2]} \frac{(-1)^{\mu}\left(\left[\frac{n-2}{2}\right]+\mu\right) !}{\left(\left[\frac{n-1}{2}\right]-\mu\right) ! \mu !} \\
& \quad \times \sum_{\lambda=0}^{k+\mu} \frac{(-1)^{\lambda_{t}[(n-1) / 2]+k-\lambda}}{(k+\mu-\lambda) ! \lambda !} .
\end{aligned}
$$

Writing $[(n-1) / 2]+k-\lambda=\rho$, we have

$$
\begin{aligned}
t^{[(n-1) / 2]} B(t) & =\frac{\left[\frac{n}{2}\right] !}{\left[\frac{n-2}{2}\right] !} \sum_{k=0}^{[n / 2]} \frac{(-1)^{k}\left(\left[\frac{n-1}{2}\right]+k\right) !}{\left(\left[\frac{n}{2}\right]-k\right) ! k !} \\
& \times \sum_{\mu=0}^{[(n-1) / 2]} \frac{(-1)^{\mu}\left(\left[\frac{n-2}{2}\right]+\mu\right) !}{\left(\left[\frac{n-1}{2}\right]-\mu\right) ! \mu !} \\
& \times \sum_{\rho=[(n-1) / 2]-\mu}^{[(n-1) / 2]+k} \frac{(-1)^{\rho} t^{\rho}}{\left(\left[\frac{n-1}{2}\right]+k-\rho\right) !\left(\mu+\rho-\left[\frac{n-1}{2}\right]\right) !} .
\end{aligned}
$$

From Lemma 2 one finds for $n \geqq 2$

$$
\begin{aligned}
t^{[(n-1) / 2]} B(t)= & \frac{\left(\left[\frac{n}{2}\right]+\left[\frac{n-1}{2}\right]\right) !}{\left[\frac{n-1}{2}\right] !\left[\frac{n-2}{2}\right] !} \\
& \times \sum_{\mu=0}^{[(n-1) / 2]} \frac{(-1)^{\mu}\left[\frac{n-1}{2}\right] ! t^{[n / 2]+[(n-1) / 2]}}{\left(\left[\frac{n-1}{2}\right]+\mu\right)\left(\left[\frac{n-1}{2}\right]-\mu\right) ! \mu !},
\end{aligned}
$$

which reduces to (23) by means of Lemma 3 . 
Dividing (23) by $t^{[(n-1) / 2]}$ one obtains

$$
B(t)=t^{[n / 2]} \text {. }
$$

Introducing (24) into (21) and using the relation

$$
2\left[\frac{n}{2}\right]+\delta=n
$$

one finds for $n \geqq 2$,

$$
I=-\sigma^{-n} \int_{0}^{1} d\left[v^{n} f_{n}(v)\right]=f_{n}(\sigma) .
$$

The cases $n=0,1$ can be verified by direct substitution of (9) in (8). This completes the proof of the following theorem.

THEOREM. Given $f_{n}(\sigma)$ on I, satisfying the conditions (a) and (b), the solution of (8) is given by (9), where $T_{n}(t), n=0,1,2, \cdots$, are Chebyshev polynomials of the first kind, and $T_{-1}(t)=T_{1}(t)$.

\section{REFERENCES}

1. Antonio Ferri, Elements of aerodynamics of supersonic flows, New York, 1949, pp. 216-221.

2. Hsue-Shen Tsien, Supersonic flow over an inclined body of revolution, J. Aero. Sci. vol. 5 (1938) pp. 480-483.

3. Th. von Kármán and N. B. Moore, The resistance of slender bodies moving with supersonic velocities with special reference to projectiles, Trans. A.S.M.E. vol. 58 (1932) pp. 303-310.

4. M. Bôcher, An introduction to the study of integral equations, London, 1929, p. 8.

CONVAIR-ASTRONAUTICS, SAN Diego and

University of California, Los ANgeles 